\title{
REALIZATION OF DIFFERENT DIVERSITY TECHNIQUE FOR MIMO BASED MOBILE AD HOC NETWORK USING SPACE TIME BLOCK CODES
}

\author{
${ }^{1}$ Swati Chowdhuri, ${ }^{1}$ Nilanjan Byabarta, ${ }^{2}$ Dr. S.S.Choudhury and ${ }^{2}$ Dr. P.K.Baneerjee \\ ${ }^{1}$ Asst.Professor, ECE, Seacom Engineering College, Howrah-711302 \\ swati.chowdhuri@gmail.com \\ nilanjan.ece@gmail.com \\ ${ }^{2}$ Professor, ETCE, Jadavpur University, kolkata-700032 \\ Pkbju65@gmail.com \\ pkbanj@rediffmail.com
}

\begin{abstract}
With the fast progress of MIMO technology and its growing applications in the mobile ad hoc networks, it is important to develop a technique to enable more efficient MIMO implemented mobile ad hoc network. The simple, yet powerful concept of an bit error rate $(B E R)$ performance calculation with respect to bit energy to noise spectral density ratio $\left(E_{b} / N_{0}\right)$ of a typical transmit diversity scheme with various number of transmit and receiving antennas. Different diversity technique for mimo based mobile ad hoc network using space time block codes are demonstrated to show the superiority in performance of MIMO over single-antenna routing schemes, particularly at high SNR.
\end{abstract}

\section{INDEX TERMS}

Space Time Block Code (STBC), Quasi-Orthogonal STBC, Bit Error Rate, Spectral Density, Maximal Ratio Combining (MRC).

\section{INTRODUCTION}

Diversity is a bandwidth efficient method for mitigating the negative effects of fading. If two or more antennas are spaced sufficiently apart the received signals in the antenna elements will fade differently. By increasing the signal quality it is possible to increase the data rate, communication range or coverage. Diversity can also be achieved by using multiple transmit antennas and by coding the signal over both space and time (or frequency), through e.g. Space-Time Block Coding (STBC) scheme [3]. Space-Time Block Code (STBC), a type of feedforward transmits diversity scheme that will be the main focus of this paper. By employing multiple transmit antennas to provide transmit diversity, the BER can be significantly reduced, such that the BER curve decays faster with $\mathrm{Eb} / \mathrm{N}$, This is due to the multiple transmit antennas providing higher spatial diversity level. However, unlike receive diversity that can be achieved by simply performing the diversity combining at the receiver side, transmit diversity requires some form of signal processing, generally known as space time coding, on the transmitted signals in order to achieve signal enhancement at the receiver. Space-Time Coding (STC) is a technique that combines coding, modulation and signal processing to achieve transmit diversity. STBC scheme supports linear decoding complexity for maximum-likelihood (ML) decoding, which is much simpler than the decoding of STTC (Space-Time Trellis Code). STBC is used for two transmit antenna whereas 0-STBC is generalizations to an arbitrary number of transmit antennas. It retains the property of having linear maximum-likelihood decoding with full transmit diversity. Although 0-STBC can provide full diversity at low computational cost, till it suffers a loss in capacity when (1) there are multiple receive antennas, (2) the code rate is less than one. 0-STBC design for more than two transmit antennas will always suffer capacity loss. To address the issue of capacity loss, various non-orthogonal STBC designs have been proposed. An interesting one among them is the Quasi-Orthogonal STBC (QO-STBC), which is designed to achieve a higher code rate than 0-STBC by partially. For example, the ML decoding of the full-rate QO-STBC for four transmit antennas can be achieved by jointly detecting two out of four complex symbols in the codeword, and separately doing the same for the remaining two complex symbols. Due to this low decoding complexity advantage of QO-STBC, as well as its ability to achieve full transmit diversity [1].

Diversity is to provide different replicas of the transmitted signal to the receiver. If these different replicas fade independently, it is less probable to have all copies of the transmitted signal in deep fade simultaneously. Therefore, the receiver can reliably decode the transmitted signal using these received signals. The probability of outage will be lower for the signal with highest SNR and receive multiple replicas of the signal using diversity. To define diversity quantitatively, we use the relationship between the received SNR, denoted by $\gamma$, and the probability of error, denoted by $P_{e}$. A tractable definition of the diversity, or diversity gain, is

$$
G_{d}=-\lim _{\gamma \rightarrow \infty} \frac{\log \left(P_{e}\right)}{\log \gamma}
$$

Where $\mathrm{P}_{\mathrm{e}}$ is the error probability at an SNR equal to $\mathrm{y}$. In other words, diversity is the slope of the error probability curve in terms of the received SNR in a log-log scale [2]. There are two important issues related to the concept of diversity. One is how to provide the replicas of the transmitted signal at the receiver with the lowest possible consumption of the power, bandwidth, decoding complexity and other resources. The second issue is how to use these replicas of the transmitted 
signal at the receiver in order to have the highest reduction in the probability of error. The replica of the transmitted signal can be transmitted in a different time slot, a different frequency, a different polarization, or a different antenna. The goal is to send two or more copies of the signal through independent fades. When different time slots are used for diversity, it is called temporal diversity. Two time intervals separated for more than the coherence time of the channel go through independent fades. Therefore, we may send copies of the transmitted signal from these separated time slots. Errorcorrecting codes can be utilized to reduce the amount of redundancy. If the fading is slow, that is the coherence time of the channel is large, the separation between time slots used for temporal diversity is high. In this case, the receiver suffers from a huge delay before it can start the process of decoding. The coded symbols are interleaved before sending through the channel. While interleaving increases the delay, it converts a slow fading channel to a fast fading channel that is more appropriate for temporal diversity. Temporal diversity is not bandwidth efficient because of the underlying redundancy. Another method of diversity is frequency diversity. Frequency diversity uses different carrier frequencies to achieve diversity. To achieve diversity, the carrier frequencies should be separated by more than the coherence bandwidth of the channel. In this case, different replicas of the signal experience independent fades. Similar to temporal diversity, frequency diversity suffers from bandwidth deficiency. Also the receiver needs to tune to different carrier frequencies. One method of diversity that may not suffer from bandwidth deficiency is spatial diversity or antenna diversity. Spatial diversity uses multiple antennas to achieve diversity. Multiple antennas may be used at the receiver or transmitter. If the antennas are separate enough, more than half of the wavelength, signals corresponding to different antennas fades independently. The use of multiple antennas may not be possible in small handheld devices. This is because of the fact that a minimum physical separation is needed between different antennas to achieve spatial diversity. Spatial diversity is not the only way to use antennas for providing diversity. Angular diversity uses directional antennas to achieve diversity. Different copies of the transmitted signal are collected from different angular directions. Another diversity method is polarization diversity that uses vertically and horizontally polarized signals to achieve diversity. Because of the scattering, the arriving signal, which is not polarized, can be split into two orthogonal polarizations. If the signal goes through random reflections, its polarization state can be independent of the transmitted polarization.

\section{MATHEMATICAL EXPRESSION OF SPACE-TIME BLOCK CODE}

Space Time Block Code can be considered as a modulation scheme for multiple transmits antennas that provide full diversity and very low complexity encoding and decoding. Before we introduce the transmit diversity scheme based on Space-Time Block Code (STBC), we first review the traditional receive diversity scheme with maximal ratio combining (MRC) for one transmit antenna and two receive antennas. For this scheme Receive diversity with one transmit and two receive antennas Denoting the transmitted signal at time $p$ as $c_{p}$, and the received signals on the first and second receive antennas are

$$
\begin{gathered}
r_{p}{ }^{(1)}=h_{1} c_{p}+n_{p}{ }^{(1)} \\
r_{p}{ }^{(2)}=h_{2} c_{p}+n_{p}{ }^{(2)}
\end{gathered}
$$

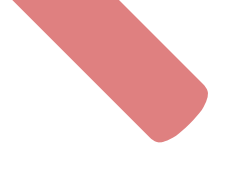

Where $h_{1}$ and $h_{2}$ are the CSI or path gain from the transmit antenna to the first and second receive antennas respectively, are additive white Gaussian noises (AWGN) at the respective received antennas at time instant $p[1,5]$. If only one receive antenna is available, the transmitted symbols can be estimated as follows, by assuming that the CSI is known accurately:

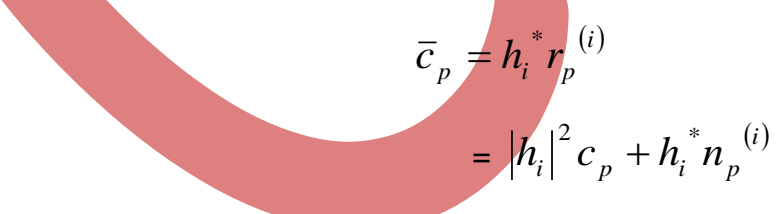

When multiple receive antennas are available, to retrieve the data symbols utilizing the diversity signals provided by multiple receive antennas, we perform MRC as follows:

$$
\begin{gathered}
\bar{c} p=h_{1}{ }^{*} r_{p}{ }^{(1)}+h_{2}{ }^{*} r_{p}{ }^{(2)} \\
=\left(\left|h_{1}\right|^{2}+\left|h_{2}\right|^{2}\right) c_{p}+h_{1}{ }^{*} n_{p}{ }^{(1)}+h_{2}{ }^{*} n_{p}{ }^{(2)}
\end{gathered}
$$

Alamouti proposed a simple two-antenna transmit diversity (STBC) scheme which maximizes to 0-STBC that achieves the same diversity gain more than two-antenna transmit diversity. Suppose during the first symbol interval, the signal s1 is transmitted from antenna 1, while s2 is transmitted from antenna 2.During the next symbol period, antenna 1 transmitted signal $s_{2}^{*}$ and antenna 2 transmit signal $s_{1}^{*}$. Thus, the signals received in two adjacent time slots are:

$$
\begin{aligned}
& r_{1}=h_{1} s_{1}+h_{2} s_{2}+n_{1} \\
& r_{2}=h_{2} s_{1}^{*}+h_{1} s_{2}^{*}+n_{2}
\end{aligned}
$$


$h_{1} h_{2}$ Denote the path gain from the two transmit antenna to the receive antennas. Spectrums of these two signals are shown below:

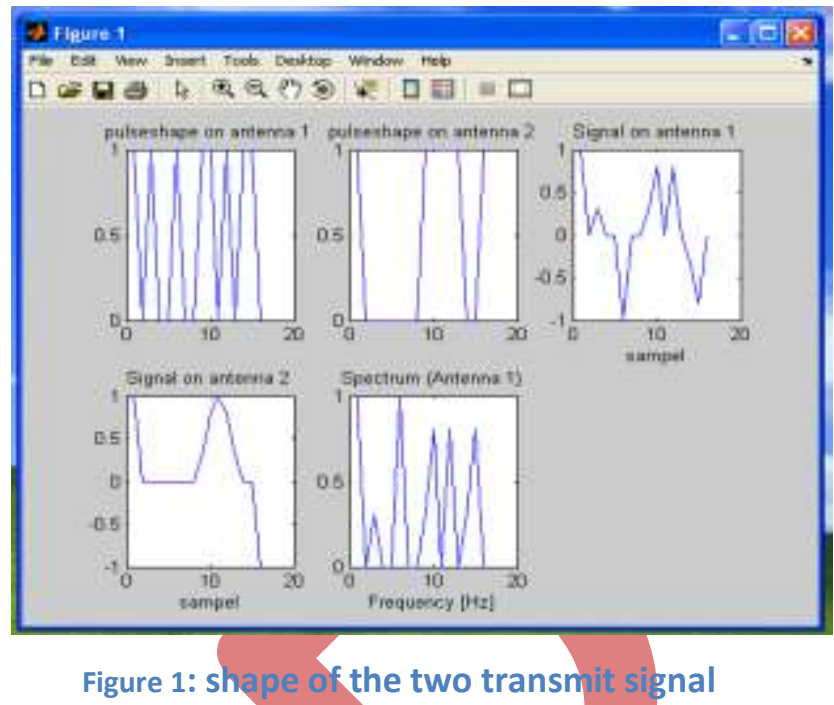

Tx antenna transmits the same power as the single antenna, the two receive antennas observe $h_{1} s_{1}+n_{1}, h_{2} s_{1}+n_{2}$. Respectively, and maximal-ratio combining the decision variable is

$$
h_{1}^{*}\left(h_{1} s_{1}+n_{1}\right)+h_{2}^{*}\left(h_{2} s_{1}+h_{2}\right)
$$

In this case, $h_{1}$ and $h_{2}$ are the CSI from the first and second transmit antenna to the single receive antenna respectively, and they are assumed to remain unchanged for two symbol periods. The received signals at time $2 p-1$ and $2 p$ can be expressed respectively as:

$$
\begin{aligned}
& r_{2 p-1}=\frac{1}{\sqrt{2}}\left(h_{1} c_{2 p-1}+h_{2} c_{2 p}\right)+\eta_{2 p-1} \\
& r_{2 p}=\frac{1}{\sqrt{2}}\left(h 2 c_{2 p-1}^{*}-h_{1} c_{2 p}^{*}\right)+\eta_{2 p}
\end{aligned}
$$

Where the factor $1 / \sqrt{2}$ accounts for the power normalization, $\eta_{2 p-1}$ and $\eta_{2 p}$ are AWGN at the receiver at time $2 \mathrm{p}-1$ and $2 p$ respectively. Assuming that perfect $\mathrm{CSI}$ is known to the receiver, the transmitted data symbols can be recovered by linear combining as shown below:

$$
\begin{gathered}
\bar{c}_{2 p-1}=h_{1} r_{2 p-1}+h_{2} r_{2 p}{ }^{*}=\frac{1}{\sqrt{2}}\left(|h|^{2}+|h|^{2}\right) c_{2 p-1} \\
+h_{1}{ }^{*} \eta_{2 p-1}+h_{2} \eta_{2 p}{ }^{*} \\
\bar{c}_{2 p}=h_{2}{ }^{*} r_{2 p-1}-h_{1} r_{2 p}{ }^{*}=\frac{1}{\sqrt{2}}\left(|h 1|^{2}+|h|^{2}\right) c_{2 p} \\
+h_{2}{ }^{*} \eta_{2 p-1}-h_{1} \eta_{2 p}{ }^{*}
\end{gathered}
$$

Therefore, the diversity order of the above 0-STBC system with simple linear receiver processing is the same as the corresponding receive diversity with MRC. Alamouti proposes O-STBC as a transmit diversity scheme that offers similar diversity gains, using multiple antennas at the transmitter. Next section highlights this comparison of transmit vs. receive diversity by simulating coherent Quadrature Amplitude modulation (QAM) over flat-fading Rayleigh channels. For transmit diversity, we use two transmit antennas and one receive antenna ( $2 \times 1$ notationally), while for receive diversity we employ one transmit antenna and two receive antennas (1 12 notationally). 
Council for Innovative Research www.cirworld.com
International Journal of Computers \& Technology

Volume 4 No. 2, March-April, 2013, ISSN 2277-3061

\section{SIMULATION AND RESULTS}

Our main aim is to minimize BER at different diversity scheme in the presence of different modulation technique. In Fig 3 a comparative study is done for transmit vs receive diversity. Plot of BER vs (Eb/N0) is shown in Fig 4 and Fig 5 for the $2 * 2$ system and $4 * 1$ systems. And finally in Fig 6 we see that the Frame Error Rate (FER) will be minimum after concatenated TCM (Trellis Coded Modulation) and OSTBC (Orthogonal Space Time Block Code).
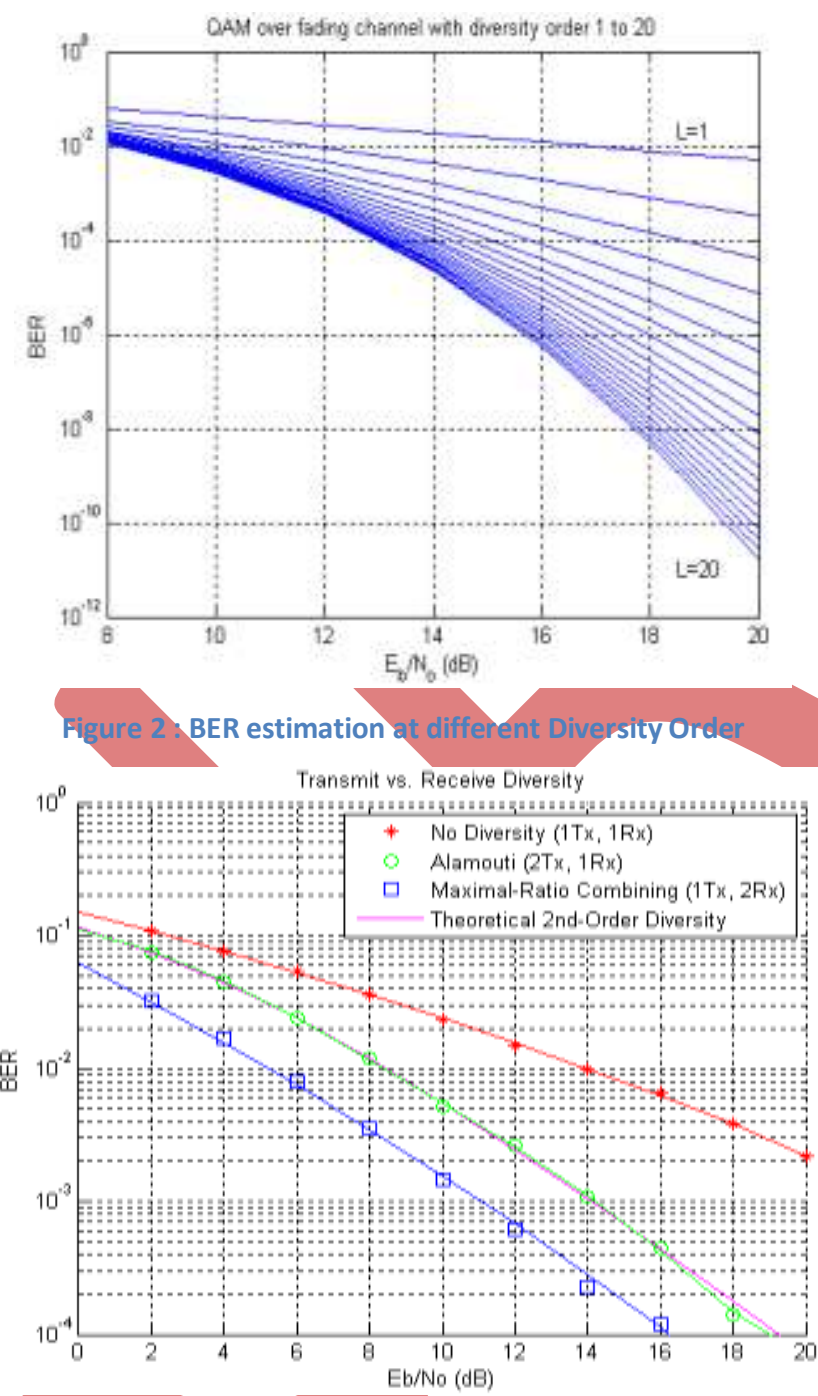

Figure 3: Comparative study of transmit and receive diversity 


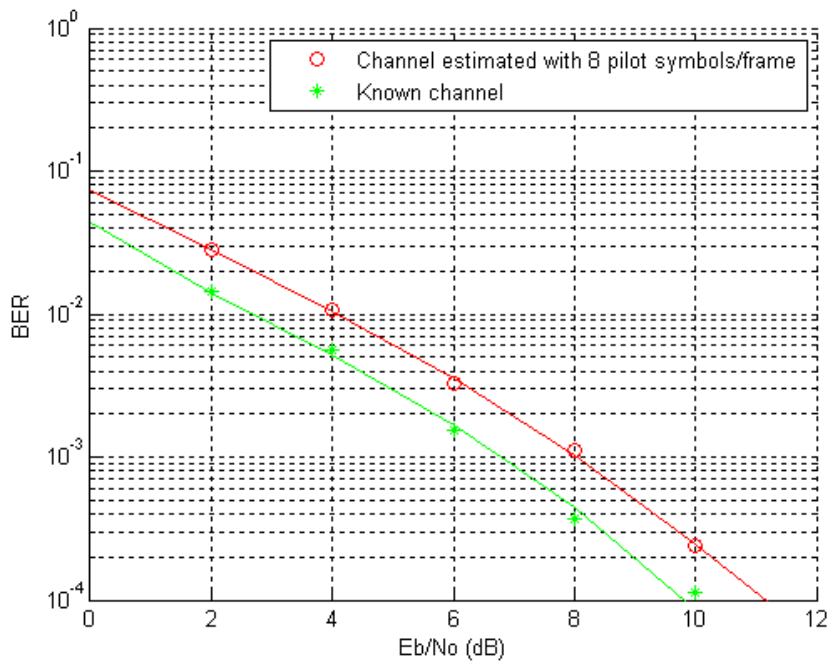

Figure 4: Eb/NO vs BER plot for $2 * 2$ system
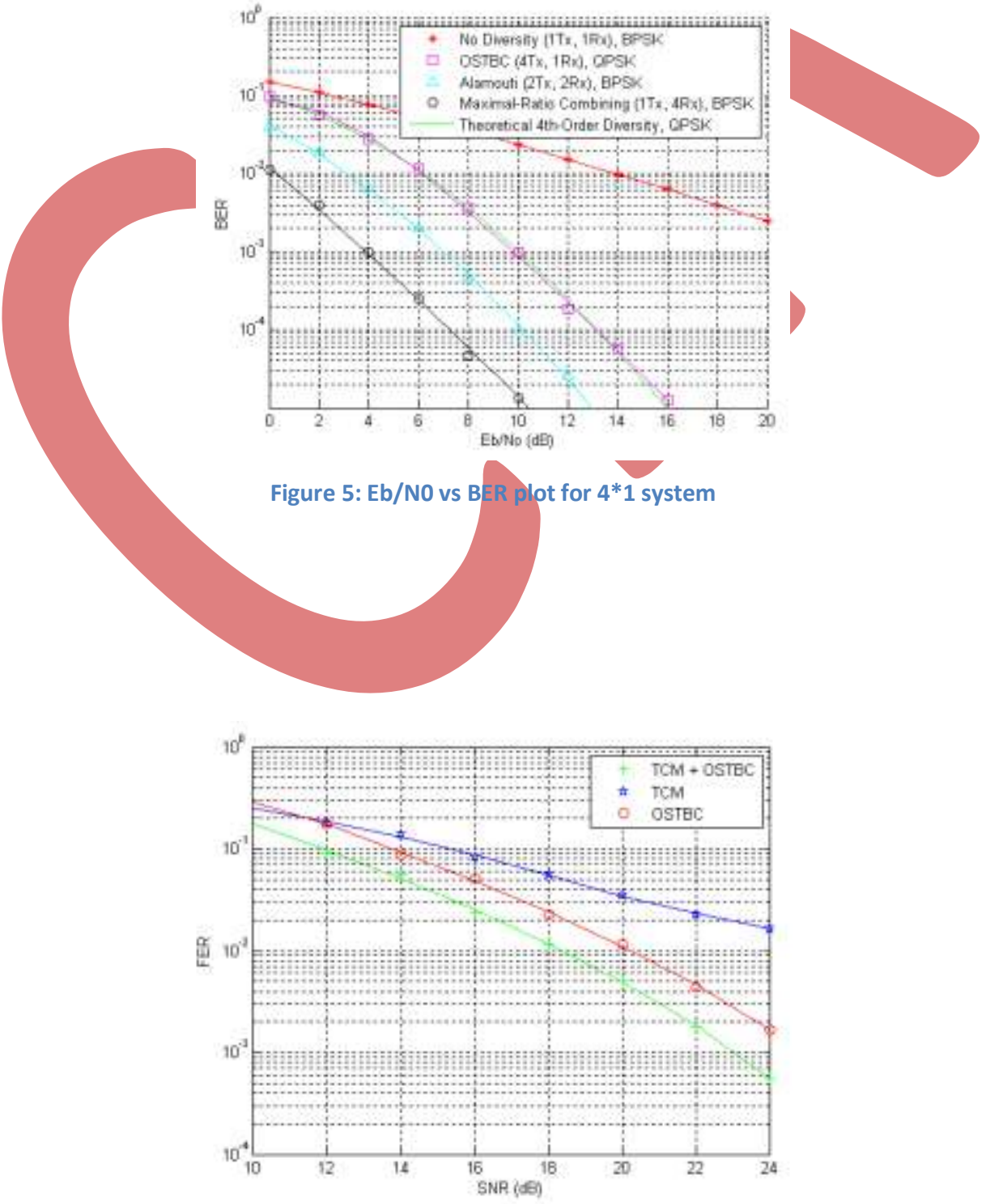


\section{CONCLUSION}

The simulation shows under various conditions, different number of transmit and receive antennas, different modulation order at the pilot and data subcarriers. Finally a comparative study of different diversity is done with the presence of different modulation scheme. STBC scheme offers an efficient and computation-effective result where the use of groups helps to improve the performance of the system without increasing the complexity at the receiver.

\section{REFERENCES}

[1] Hemanth Sampath, "Linear Precoding and Decodng for Multiple Input Multiple Output (MIMO) Wireless Channels", stanford university, April 2001.

[2] Mr. Chaiyod Pirak, "Spatial Interference Cancellation and Channel Estimation for Multile-Input-Multiple-Output Wireless Communication Systems, Chulalongkorn University, ISBN 974-53-2403-5, 2005.

[3] Jing Xiao, ANALYSIS OF OFDM MIMO.

[4] Daniel Aronsson. "Channel Estimation and Prediction for MIMO OFDM Systems Key Design and Performance Aspects of Kalman-based Algorithms".

[5] Shan Chu, Xin Wang. "Opportunistic and Cooperative Spatial Multiplexing in MIMO Ad hoc Networks".

[6] W. Ajib, D. Haccoun, An overview of scheduling algorithms in MIMO- based fourth-generation wireless systems, IEEE Network, Volume 19, Issue 5, pp. 43 - 48, Sept.-Oct. 2005.

[7] V.K.N., Lau, Y. Liu, T. A. Chen, Optimal multi-user space time scheduling for wireless communications, Proc. IEEE VTC 2002-Fall, Volume 4, pp. 1939- 1942, 2002.

[8] L. Bao and J.J. Garcia-Luna-Aceves, "Transmission Scheduling in Ad Hoc Networks with Directional Antennas", Proc. ACM/IEEE MobiCom 2002.

[9] Sunil Srinivasa, Martin Haenggi, "Simplified Analysis and Design of MIMO Ad Hoc Networks".

[10] Fabien Delestre, "Channel Estimation and Performance Analysis of MIMO-OFDM Communications using SpaceTime and Space-Frequency Coding Schemes", STRI, U.K., July 2011.

[11] Zhou Wen ,"A Study of Channel Estimation for OFDM Systems and System Capacity for MIMO-OFDM Systems" ,2010 http://hdl.handle.net/10722/132954.

[12] Nadew Adisu, "Low Complexity MIMO-OFDM Receivers for Achieving Near Optimal Performance", ADDI ABABA UNIVERSITY, July, 2011.

Swati Chowdhuri: Presently, she is an Asst.Professor of Seacom Engineering College. She has completed B.E. in Electronics and communication Engg from Burdwan University with Honours in the year of 2005 and completed M.Tech in Computer Technology from Jadavpur University in the year of 2009. Her research interests in the field of "MIMO based Mobile Ad-hoc networks".

Nilanjan Byabarta: Presently, he is an Asst.Professor of Seacom Engineering College. He has completed B.Tech.in Electronics and communication Engg. from West Bengal University of Technology with Honours in the year of 2008 and completed M.Tech in V.L.S.I Design and Microelectronics Technology from Jadavpur University in the year of 2012. His research interests in the field of "MIMO based Mobile Ad-hoc networks" and "Cognitive Radio" 